\title{
Untangling the Dynamic Structure of an Enterprise by Applying a Fractal Approach to Business Processes
}

\author{
Ilia Bider, Erik Perjons, and Mturi Elias \\ DSV, Stockholm University, Forum 100, 16440 Kista, Sweden \\ \{ilia, perjons, mturi\}@dsv.su.se
}

\begin{abstract}
A promising approach for analyzing and designing an enterprise is to consider it as a complex adaptive system (CAS) able to self-adjust to the changes in the environment. An important part of designing a CAS model is to untangle the dynamic structure of an enterprise. This paper presents a procedure for identifying all processes that exist in an enterprise as well as their interconnections. The procedure makes use of a number of process-assets and asset-processes archetypes. The first ones help to find out what assets are needed for a particular process, the second ones help to find out supporting processes that are needed to have each type of assets ready available for deployment. The procedure is based on the ideas of fractal organization where the same pattern is repeated on different levels. The uncovered dynamic structure of an enterprise can support strategic planning, change management, as well as discovering and preventing misbalances between its business processes. The paper also presents an example of applying the procedure to research activities of a university.
\end{abstract}

Keywords: Business Process, Enterprise Modeling, Fractal Enterprise.

\section{Introduction}

One of the main characteristics of the environment in which a modern enterprise functions is its high dynamism due to globalization and speedy technological progress. To survive and grow in the dynamic environment with global competition for customers, capital and skilled workforce, a modern enterprise should be able to quickly adapt itself to changes in the environment, which includes using opportunities these changes offer for launching new products and services.

This new enterprise environment has already attracted attention of researchers who started to consider an enterprise as a complex adaptive system (CAS) able to selfadjust to the changes in the environment [1-4]. The long-term goal of our research project is to create a practical methodology for modeling an enterprise as a multilayered CAS capable of self-adaptation without centralized planning mechanism. Building such a model requires finding interconnections between various components of the enterprise. Such interconnections should allow efficient information exchange between the layers so that changes in various parts of the enterprise environment are promptly discovered and dealt with. The objective of having such a model is to help 
an enterprise to better understand its existing structure so that it could be fully exploited and/or improved.

In the short term, our research is currently focused on getting answers to the following two interconnected questions:

- How to find all processes that exist in an enterprise? This is not a trivial matter as only most visible processes catch attention of management and consultants. These processes represent only the tip of an iceberg of what exists in the enterprise in half-documented, or in totally undocumented form (tacit knowledge).

- What types of interconnections exist between different business processes and how they can be represented in an enterprise model? The answer is needed to get a holistic view on the enterprise processes which is one of the objectives of having an enterprise model.

Besides helping to achieve our long terms goals, such answers, if found, have their own practical application. Without knowing all business processes and their interconnections, it is difficult to plan any improvement, or radical change. Changes introduced in some processes without adjusting the associated processes may have undesirable negative consequences. Having a map of all processes and their connections could help to avoid such situations.

This paper is devoted to finding answers to the above two questions. This is done based on the enterprise model from [5] that represents an enterprise as consisting of three types of components: assets (e.g., people, infrastructure, equipment, etc.), sensors and business process instances. The working hypothesis, when answering the questions above, is that the processes and their relationships can be uncovered via the following procedure. One starts with the visible part of the iceberg, so-called main processes. Here, as main we count processes that produce value for which some of the enterprise external stakeholders are ready to pay, e.g., customers of a private enterprise, or a local government paying for services provided to the public. Typical examples of main processes are hard (e.g., a computer) or soft (e.g., software system) product manufacturing, or service delivery (e.g., educational process at a university). When the main processes are identified, one proceeds "under water" following up assets that are needed to run the main processes. Each assets type requires a package of so-called supporting processes to have the corresponding assets in "working order" waiting to be deployed in the process instances of the main process. To supporting processes belong, for example, human resources (HR) processes (e.g., hiring or retiring members of staff) that insure the enterprise having right people to be engaged in its main processes.

To convert the working hypothesis above into a procedure that could be used in practice, we introduce:

- Process-assets archetypes (patterns) that help to find out what assets are needed for a particular process, especially for a main process from which we start unwinding,

- Assets-processes archetypes (patterns) that help to find out supporting processes that are needed to have each type of assets ready available for deployment. 
Having these archetypes/patterns will help us to unveil the dynamic process structure of an enterprise starting from the main process and going downwards via repeating pattern "a main process->its assets->processes for each assets->assets for each process-> ...". As the result we will get an indefinite tree consisting of the same type of elements. Such kind of structures is known in the scientific literature under the name of fractal structures [6].

Based on the deliberations above, the goal of this paper is to introduce the processassets and asset-processes archetypes/patterns, and show how to use them in practice to untangle the dynamic structure of an enterprise. The example we use for the latter is from the academic world. We start from one of the main processes - research project - in the university world and unwind it according to the procedure outlined above. The example was chosen based on the authors having their own experience of this process type as well as easy access to the expertise of the colleagues. The chosen example does not mean that the procedure is applicable only to the university world. When discussing the archetypes, we will give examples from other types of enterprises as well.

The research presented in the paper is done in the frame of the design science paradigm [7,8]. The goal of such kind of research is finding and testing a generic solution [8], or artifact in terms of [7], for a class of practical problems. The archetypes and procedure of using them suggested in the paper constitutes a design science artifact for getting an answer for the two main questions discussed. Though most of the concepts used in building this artifact are not new, the artifact itself, which is the main contribution of the paper, as a whole is new and original. In addition, we do not know any research work specifically devoted to finding answers to the questions above. So our solution, even if not perfect, can be used in practice until a better one could be found.

The rest of the paper is structured in the following way. In Section 2, we present an overview of our three-layered enterprise model from [5]. In Section 3, we discuss process and assets archetypes (patterns). In section 4, we apply these patterns to unwind parts of the dynamical structure of a university. In Section 5, we discuss some related works. Section 6 discusses the results achieved and plans for the future.

\section{The Assets-Sensors-Processes Model of an Enterprise}

Our starting point is a systemic approach to the enterprise modeling from [5]. We consider an enterprise as a system that reacts on different situations constantly emerging in its environment or inside itself to maintain the balance between itself and environment or inside itself. An emerging situation is dealt by creating a respondent system [9] that is disbanded after the situation has been dealt with. The respondent system is built from the assets that the larger system already has. Some of these assets are people, or other actors (e.g., robots). Other assets are control elements, e.g., policy documents, which define the behavior of the respondent system.

To deal with emerging situations effectively, an enterprise creates templates for the majority of known types of situations. Such a template is known under different 
names, like project template, business process definition, business process type, or business process model. We will refer to it as to Business Process Template (BPT). BPT contains two parts:

1. Start conditions that describe a situation which warrants creation of a respondent system

2. Execution rules that describe a composition and behavior of a respondent system

A respondent system created according to the BPT template has different names, e.g., a project or a case. We will refer to such a system as to Business Process Instance (BPI).

Note that PBTs can exist in an organization in an explicit or implicit form, or a combination of both. Explicit BPTs can exist as written documents (e.g. employee's handbooks or position descriptions), process diagram, or built in computerized system that support running BPIs according to the given BPTs. Implicit BPTs are in the head of the people engaged in BPIs that follows given BPTs. These BPTs belongs to what is called tacit knowledge.

Based on the systemic view above, we consider an enterprise as consisting of three types of components, assets, sensors and BPIs, depicted in Fig. 1, and explained below:

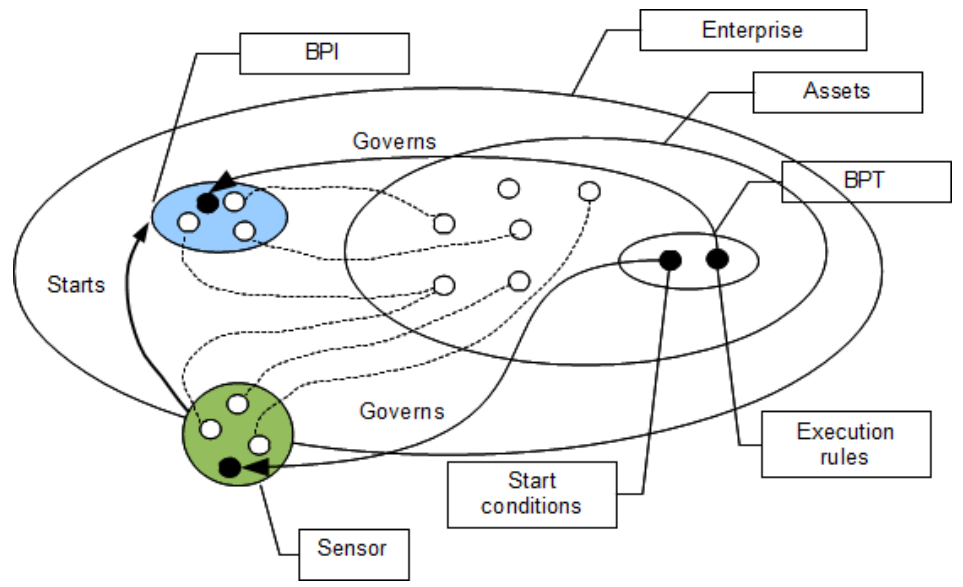

Fig. 1. An enterprise model consisting of three types of components: assets, sensors and BPIs

1. Assets (tangible and intangible):

- People with their knowledge and practical experiences, beliefs, culture, sets of values, etc.

- Physical artifacts - computers, telephone lines, production lines, etc.

- Organizational artifacts, formal as well as informal - departments, teams, networks, roles, etc.

- Information artifacts - policy documents, manuals, business process templates (BPTs), etc. To information artifacts belong both written (documented) artifacts, and tacit artifacts - the ones that are imprinted in the people's heads (e.g., culture) 
The assets are relatively static, which means that by themselves they cannot change anything. Assets are activated when they are included in the other two types of components. Assets themselves can be changed by other types of components when the assets are set in motion for achieving some goals. Note that assets here are not regarded in pure mechanical terms. All "soft" assets, like sense of common goals, degree of collaborativeness, shared vision, etc., belong to the organizational assets. Note also that having organizational artifacts does not imply a traditional function oriented structure. Any kind of informal network or resource oriented structural units are considered as organizational artifacts.

2. Sensors are a set of (sub)systems, the goal of which is to watch the state of the enterprise itself and its environment and catch impulses and changes (trends) that require firing of BPIs of certain types. We need a sensor (which might be a distributed one) for each BPT. The work of a sensor is governed by the Start Conditions of the BPT description (which is an informational artifact). A sensor can be fully automatic for some processes (an order placed by a customer in a webbased shop), or require human participation to detect changes in the system or its surroundings.

3. BPIs - a set of respondent systems initiated by sensors for reaching certain goals and disbanded when these goals are achieved. The behavior of a BPI system is governed by the Execution Rules of the corresponding BPT. Dependent on the type, BPIs can lead to changes being made in the assets layer. New people are hired or fired, departments are reorganized, roles are changed, new policies are adopted, BPT descriptions are changed, new BPTs are introduced, and obsolete ones are removed.

\section{Process-Assets and Asset-Processes Archetypes}

In [5], we have discussed several types of interrelationships between the components of an enterprise overviewed in the previous section, namely:

1. Sensors and BPIs use assets to complete their mission: to discover needs for fire a BPI for a sensor, or to attain a goal for BPI.

2. BPIs can change the assets

3. A sensor, as well as BPI can be recursively decomposed using the assets-sensorsprocesses model of Fig. 1.

In this paper, we concentrate only on the first two types of relationships between the components of the enterprise, leaving the third type, process decomposition, outside the scope of this paper. In other words, we will not be discussing any details of the internal structure of processes, focusing only on what types of assets are needed for running process instances of a certain type and in what way process instances can affect the assets. 


\subsection{The Process-Assets Archetype for Main Processes}

We consider as enterprise any organization the operational activities of which are financed by external stakeholders. It can, for example, be a private company that gets money for its operational activities from the customers, a head office of an interest organization that gets money from the members, or a public office that gets money from the taxpaying citizens or inhabitants. We consider a main (or core) process to be a process that produces value to the enterprise's external stakeholders for which they are willing to pay. Our definition of the term main (or core) process may not be the same as those of others $[10,11]$. For example, we consider as main processes neither sales and marketing processes, nor product development processes in a product manufacturing company. However, our definition of the main process does cover processes of producing and delivering products and services for external stakeholders, which is in correspondence with other definitions of main processes [10,11].

Main processes are the vehicles of generating money for operational activities. To get a constant cash flow, an enterprise needs to ensure that new business process instances (BPIs) of main processes are started with some frequency. To ensure that each started BPI can be successfully finished, the enterprise needs to have assets ready to be employed so that the new BPI gets enough of them when started. We consider that any main process requires the following six types of assets (see also Fig. 2 and 3):

1. Paying stakeholders. Examples: customers of a private enterprise, members of an interest organization, local or central government paying for services provided for the public. ${ }^{1}$

2. Business Process Templates (BPTs). Examples are as follows. For a production process in a manufacturing company, BPT includes product design and design of a technological line to produce the product. For a software development company that provides customer-built software, BPT includes a software methodology (project template) according to which their systems development is conducted. For a service provider, BPT is a template for service delivery.

3. Workforce - people trained and qualified for employment in the main process. Examples: workers at the conveyor belt, physicians, researchers.

4. Partners. Examples: suppliers of parts in a manufacturing process, a lab that complete medical tests on behalf of a hospital. Partners can be other enterprises or individuals, e.g., retired workers that can be hired in case there is temporal lack of skilled workforce to be engaged in a particular process instance.

5. Technical and Informational Infrastructure - equipment required for running the main process. Examples: production lines, computers, communication lines, buildings, software systems etc.

6. Organizational Infrastructure. Examples: management, departments, teams, policies regulating areas of responsibilities and behavior.

Below we give some additional clarification on the list of assets above.

\footnotetext{
${ }^{1}$ In some works all paying stakeholders are considered as customers. We prefer to differentiate these two terms as not to be engaged in the discussions not relevant to the issues of this paper.
} 
- The order in which the asset types are listed is arbitrary, and does not reflect the importance of assets of a given type; all of them are equally important.

- Our notion of asset does not coincide with the one accepted in the world of finance [12]. Except the technical infrastructure, all assets listed above belong to the category of so-called intangible assets of the finance world. Intangible assets usually lack physical substance and their value is difficult to calculate in financial terms. Technical infrastructure belongs to the category of fixed (i.e., having physical substance) tangible (i.e., the value of which is possible to calculate in financial terms) assets.

- All of the following three types of assets - paying stakeholders, skilled workforce, and partners - belong to the category of stakeholders. We differentiate them by the role they play in the main business processes. Paying stakeholders, e.g., customers, pay for the value produced in the frame of process insatnces. Workforce directly participates in the process instances and get compensation for their participation (e.g., in the form of salary). Partners provide the process with resources needed for process instances to run smoothly, e.g., electricity (power provider), money (banks or other type of investors), parts, etc. Partners get compensation for their products and services in form of payment, profit sharing, etc.

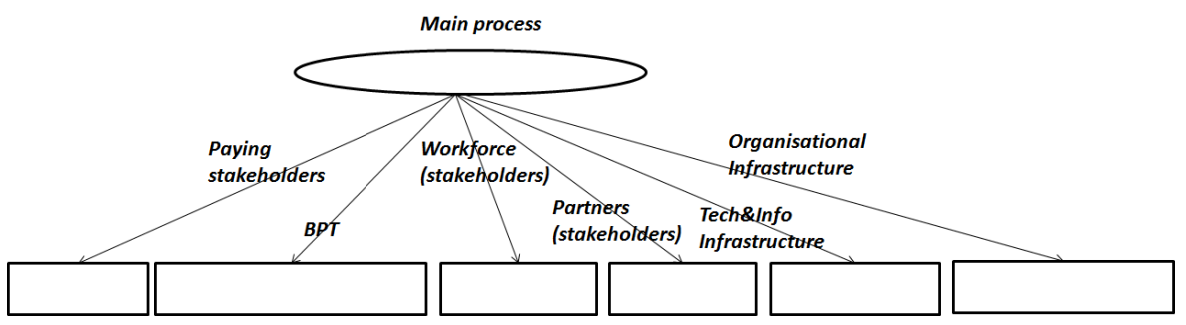

Fig. 2. The process-assets archetype for main processes

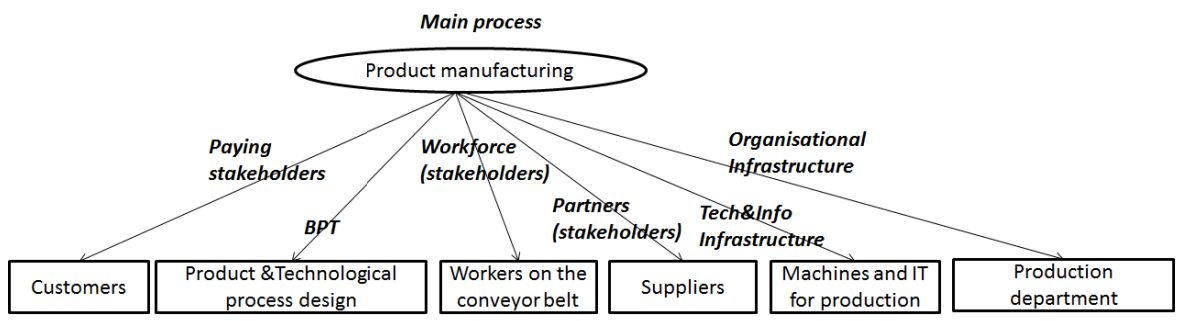

Fig. 3. An example of instantiation of the process-assets archetype for main processes

The type of processes (main) together with types of assets required for running it constitute a process-assets archetype ${ }^{2}$ for main processes. Graphically it is depicted in the form of Fig. 2, in which the process type is represented by an oval and assets

${ }^{2}$ In this paper, we use term archetype in its general meaning of "the original pattern or model of which all things of the same type are representations or copies", and not as a pattern of behavior as is widely accepted in Systems Thinking literature. 
types - by rectangles. An arrow from the process to an asset shows the needs to have this type of assets in order to successfully run process instances of the given type. A label on an arrow shows the type of assets. Instantiation of the archetype is done by inserting labels inside the oval and rectangles. Fig. 3 is an example of such instantiation for a product manufacturing process.

\subsection{The Asset-Processes Archetype}

In Section 3.1, we have introduced six types of assets that are needed to ensure that BPIs of a main process run smoothly and with required frequency. Each assets type requires a package of supporting processes to ensure that it is in condition ready to be employed in BPIs of the main process. We present this package as consisting of three types of processes connected to the life-cycle of each individual asset (see also an example in Fig. 4):

1. Acquire - processes that result in the enterprise acquiring a new asset of a given type. The essence of this process depends on the type of asset, the type of the main process and the type of the enterprise. For a product-oriented enterprise, acquiring new customers (paying stakeholders) is done through marketing and sales processes. Acquiring skilled work force is a task completed inside a recruiting process. Acquiring a new BPT for a product-oriented enterprise is a task of new product and new technological process development. Creating a new BPT also results in introducing a new process in the enterprise.

2. Maintain - processes that help to keep existing assets in right shape to be employable in the BPIs of a given type. For customers, it could be Customer Relationship Management (CRM) processes. For workforce, it could be training. For BPT, it could be product and process improvement. For technical infrastructure, it could be service.

3. Retire - processes that phase out assets that no longer can be used in the main process. For customers, it could be discontinuing serving a customer that is no longer profitable. For BPTs, it could be phasing out a product that no longer satisfies the customer needs. For workforce, it could be actual retirement.

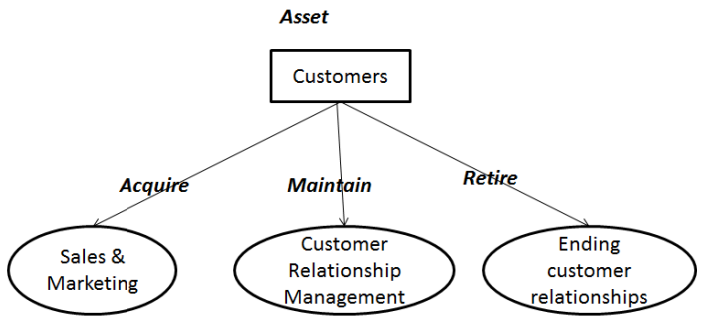

Fig. 4. An example of instantiation of the asset archetype

The asset-processes archetype can be graphically presented in the form of Fig. 4. In it, the asset type is represented by a rectangle, and a process type - by an oval. An arrow from the asset to a process shows that this process is aimed at managing assets 
of the given type. The label on the arrow shows the type of the process - acquire, maintain, or retire. Instantiation of the archetype is done by inserting labels inside the rectangle and ovals. Actually, Fig. 4 is an example of such instantiation for the customer's assets in a manufacturing company (on the difference between archetypes and instantiations, see Fig. 2 and 3 and the text related to them in Section 3.1).

\subsection{Archetypes for Supporting Processes}

Types of assets that are needed for a supporting process can be divided into two categories, general asset types, and specific ones. General types are the same as for the main process, except that a supporting process does not need paying stakeholders. The other five types of assets needed for a main process: BPT, workforce, partners, technical and informational infrastructure, organizational infrastructure, might be needed for a supporting process as well.

Note also that some supporting processes, e.g., servicing a piece of infrastructure, can be totally outsourced to a partner. In this case, only the partner's rectangle will be filled when instantiating the archetype for such a process.

Additionally to the five types of assets listed above, other types of assets can be added to a specific category of supporting processes. We have identifying two additional assets for supporting processes of acquiring an asset that belongs to the category of stakeholders, e.g., paying stakeholders, workforce, and partners:

- Value proposition, for example, description of products and/or services delivered to the customer, or salary and other benefits that an employee gets.

- Reputation, for example, of being reliable vendor, or being a great place of work.

Adding the above two asset types to the five already discussed, gives us a new process-assets archetype, i.e., the archetype for the acquiring stakeholders. An example of instantiation of such an archetype is presented in Fig. 5. There might be other specific archetypes for supporting processes, but so far we have not identified any more of them.

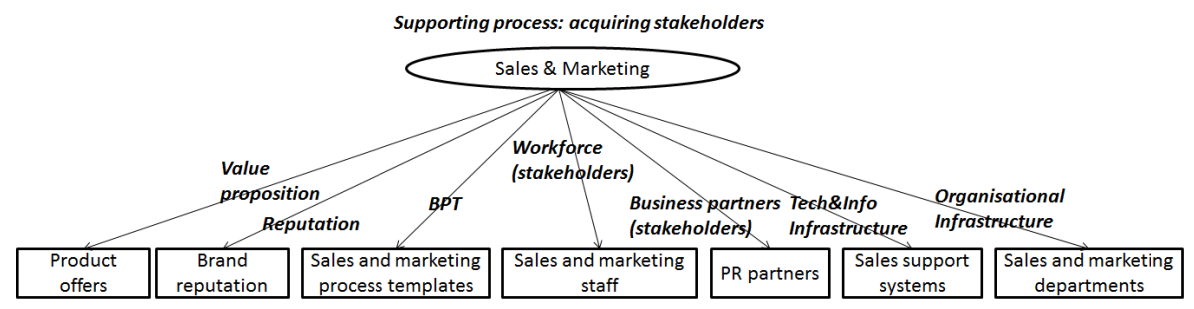

Fig. 5. An example of instantiation of the process-assets archetype for acquiring stakeholders

\subsection{Harnessing the Growth of the Processes-Assets Tree}

Using archetypes introduced above, we can unwind the process structure of the enterprise. Potentially the resulting tree will grow down and in breadth indefinitely. 
As an enterprise has a limited size, there should be some mechanisms that contain this growth and, eventually, stops it. We see several mechanisms that harness the growth:

- Some processes, e.g., maintenance of infrastructure, can be outsourced to a partner. In this case, only the partner part of a corresponding archetype will be filled.

- Some processes can share assets, e.g., workforce and BPT. For example, recruiting of staff can be done according to the same template and by the same employees working in the HR department independently whether the recruitment is done for the employees of main or supporting processes.

- Some processes can be used for managing more than one asset. For example, the assets Product offers from Fig. 5 (Value proposition asset) and Product\&Technological process design from Fig. 3 (BPT asset) are to be acquired by the same process of New product development. There is too tight interconnection between these two assets so that they cannot be created separately, e.g.:

- The offers should be attractive to the customer, so the product should satisfy some customer needs

- The price should be reasonable, so the technological process should be designed to ensure this kind of a price

- A process on an upper level of the tree can be employed as a supporting process on the lower level, which terminates the growth from the corresponding node. For example, one of the "supporting" processes for acquiring and maintaining the asset Brand reputation from Fig. 5 is the main production process itself which should provide products of good quality.

\section{$4 \quad$ Testing the Model}

The archetypes introduced in Section 3 were obtained by abstracting known facts about the structure and functioning of a manufacturing company. Therefore, testing the ideas should be done in a different domain. We choose to apply the model to an academic "enterprise", more exactly, we start unwinding the Research project process. The result of applying the process-assets archetype from Fig. 2 to this process is depicted in Fig. 6.

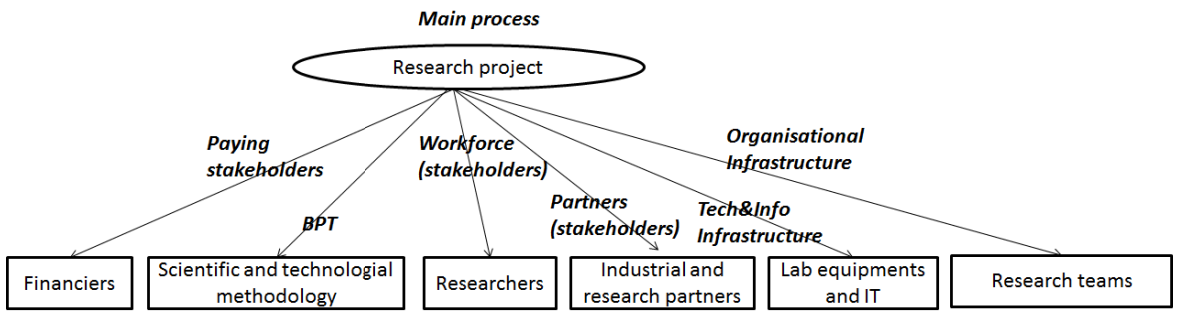

Fig. 6. Instantiation of the process-assets archetype for the main process: Research project 
The main difference between Fig. 3, which instantiates product manufacturing, and Fig. 6 is that Research project has financiers rather than customers as paying stakeholders. The result of a research process is new knowledge that is accessible for everybody, but is financed by few, including private donors who might not directly benefit from their payments. Financiers can be of several sorts:

- Research agencies giving grants created by local or central governments, or international organizations

- Industrial companies that are interested in the development of certain areas of science

- Individuals that sponsors research in certain areas

Let us consider that a financier is a research agency giving research grants. Then, applying the asset-processes archetype from Section 3.2 to the leftmost node (Financiers) of Fig. 6, we get an instantiation of this archetype depicted in Fig. 7.

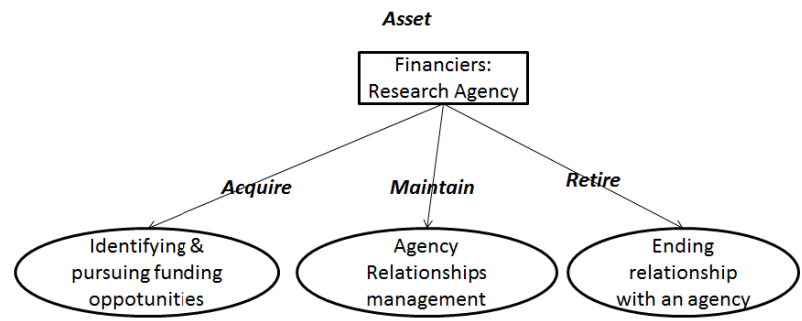

Fig. 7. Instantiation of the assets-processes archetype for a financier Research agency

Applying the Acquiring the stakeholders archetype from Section 3.3 to the leftmost node of Fig. 7 (Identifying \& pursuing funding opportunities), we will get its instantiation depicted in Fig. 8 (only the first four assets are presented in this figure).

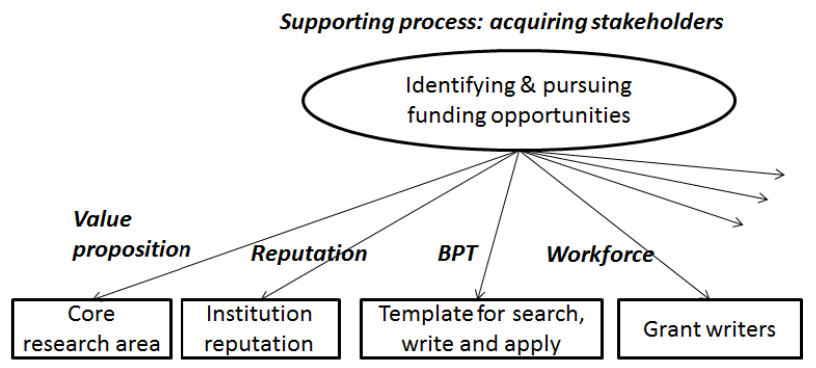

Fig. 8. Instantiation of the Acquiring stakeholders archetype to Identifying and pursuing funding opportunities

We made an experiment of interviewing two research team leaders in our institution based on Fig. 6, 7, 8. They managed to identify their core research areas and what kind of reputation they use when applying for grants. This took some time, 
as they did not have explicit answers ready. They also noted that the model helps to better understand the supporting processes around their research work. This experiment, albeit limited, shows that the model can be useful in understanding the dynamic structure of an enterprise. However, more experiments are required to validate the usefulness of our approach.

\section{$5 \quad$ Related Research}

Analysis of enterprises based on the idea of fractality has been done by several researchers and practitioners, e.g., [4], [13], [14], [15]. Their approaches differ from that of ours, which comes as no surprise as there is no accepted definition of what fractals mean in respect to the enterprise world. In essence, fractals are a high-level abstract idea of a structure with a recurring (recursive) pattern repeating on all levels. Dependent on the perspective chosen for modeling of a real life phenomenon, this pattern will be different for different modelers. Below, due to the size limitations, we only shortly summarize the works on fractal structures in enterprise modeling, and show the difference between them and our approach.

The book of Hoverstadt [13] uses the viable system model (VSM) to unfold the fractal structure of the enterprise via the system - subsystems' relationships. Subsystems are considered as having the same structure and generic organizational characteristics as the system in which they are enclosed. The resulting structure helps to analyze whether there is a balance between the subsystems. Overall, our long term goal is similar to Hoverstadt's: create a methodology for modeling an enterprise as a multilayered complex adaptive system. However, we use a completely different approach to enterprise modeling, instead of system subsystems relationships, we interleave processes and assets when building an enterprise model.

Another approach to analysis of enterprise models based on the idea of fractality can be found in Sandkuhl \& Kirikova [14]. The idea is to find fractal structures in an enterprise model built when using a general modeling technique. [14] analyzes two such models in order to find fractals in it. The results are mixed, some fractals are found, but the suspicion that many others are missed remains, due to they may not be represented in the models analyzed. The approach in [14] radically differs from that of ours. We have a hypothesis of a particular fractal structure to be found when analyzing an enterprise, while [14] is trying to find any types of the fractal structures based on the generic characteristics of organizational fractals.

Canavesio and Martinez [15] presents a conceptual model for analyzing a fractal company aiming at supporting a high degree of flexibility to react and adapt quickly to environmental changes. Main concepts are project, resource, goal, actors, plan, and relationships thereof. The approach from [15] differs from that of ours in the kind of fractals used for enterprise modeling. Fractals from [15] concern the detailed structure of business processes, while we are looking only on the relationships between processes and assets.

The focus on process organization when applying fractal principles can be found in [4]. [4] is using a pattern of sense-and-respond processes on different organizational levels each consisting of the same pattern: requirement, execution and delivery. 
The difference between our approach and that from [4] is the same as we have mentioned above. [4] is looking at the details of individual processes, we are trying to catch general relationships between different processes.

\section{Discussion and Future Research}

This paper suggests a new type of enterprise modeling that connects enterprise processes in a tree-like structure where the main enterprise processes serve as a root of the tree. The tree expands via finding all assets needed for smooth functioning of the main processes, and after that, via finding all supporting processes that are needed to handle these assets. The tree has a recursive/fractal form, where instantiations of process-assets archetypes are interleaved with those of asset-processes archetypes.

We see several practical areas where a model connecting all processes and assets in an enterprise could be applied, e.g.:

- As a help in strategic planning for finding out all branches of the processes-assets tree that require adjustments. For example, when sales plans a new campaign that will bring new customers, all assets required by the corresponding main process should be adjusted to satisfy the larger number of customers. This includes workforce, suppliers, infrastructure, etc. The calculation itself can be done with one of the known Systems Thinking methods, e.g., Systems Dynamics.

- To prevent "organizational cancer" as described in [13], p 57, when a supporting process starts behaving as it were a main one disturbing the balance of the organizational structure. This is typical for IT-departments that may start finding external "customers" for software developed for internal needs.

- As a help in radically changing the direction. When all supporting processes are mapped in the tree, it will be easier for the enterprise to change its business activity by picking up some supporting processes and converting it to the main one, while making appropriate adjustments to the tree. For example, a product manufacturing company could decide to become an engineering company. Such a decision can be made when manufacturing becomes unprofitable, while the company still have a very strong engineering department. An example of such transformation is described in [13], p. 74. Another example comes from the experience of the first author who worked for an US company that made such transformation twice. First transformation was from being a software consulting business to becoming a software product vendor when the consulting business could not accommodate the existing workforce. The second time it was done in a reverse order when a market for their line of products suddenly collapsed.

As far as future research is concerned, we plan to continue our work in several directions:

- Continuing testing. The model presented in this paper has been tested only in a limited scope, and it requires further testing and elaboration. The next major step in our research is to build a full tree with Research project as a root. This will help us to further elaborate the model, and improve our catalog of process archetypes. 
Furthermore, we need to test this modeling technique in another domain, for example, to build a model for a software development company.

- Continuing working on the graphical representation of the model. Two aspects need to be covered in this respect:

- Representing multiplicity, e.g., multiple and different assets of the same kind that require different supporting process.

- Representing sharing assets and processes in the model as discussed in section 3.4 .

- Using the processes-assets model as a foundation for modeling and designing an enterprise as a CAS (complex adaptive system). Different processes discovered with the procedure suggested in this paper are connected to different parts of the external and/or internal environment of the enterprise. If participants of these processes are entrusted to watch and report on changes in their parts of the environment, it could create a set of effective sensors (see Section 2) covering all aspects of the enterprise environment. Connecting these sensors to firing adaptation processes will close the "adaptation loop". As an example for the above, assume that the recruiting process shows that it becomes difficult to recruit skilled workforce for a main process. This fact can fire an investigative process to find out the reason for these difficulties. It could be that nobody is willing to learn such skills any more, or the competitors are expanding and offer better conditions (e.g., salary), or the enterprise reputation as a good place of work has been shattered. Based on the result of the investigation appropriate changes can be made in HR processes themselves or in completely different parts of the enterprise.

- Another application area of our processes-assets model is analyzing and representing process models in a repository. As pointed in [16], an attractive alternative to designing business processes from scratch is redesigning existing models. Such an approach requires the use of process model repositories that provide a location for storing and managing process knowledge for future reuse. One of the key challenges of designing such a repository is to develop a method to analyze and represent a collection of related processes [17]. The process-assets and asset-processes archetypes provide a mechanism to analyze and represent the relationships between business processes in a repository. The processes-assets relationships structure, when represented in the repository, will serve as a navigation structure that determines the possible paths for accessing process models by imposing an organized layout on the repository's content.

Acknowledgements. We are grateful to our colleagues, Paul Johannesson, Hercules Dalianis and Jelena Zdravkovic who participated in interviews related to the analysis of the research activity reported in Section 4. We are also thankful to David Alman, Gene Bellinger, Patrick Hoverstadt, Harold Lawson and anonymous reviewers whose comments on the earlier draft of this paper helped us to improve the text. 


\section{References}

[1] Piciocchi, P., Bassano, C., Kirikova, M., Makna, J., Stecjuka, J.: Managing Change in Fractal Enterprises and IS Architectures from a Viable Systems Perspective. In: Niedrite, L., Strazdina, R., Wangler, B. (eds.) BIR 2011 Workshops. LNBIP, vol. 106, pp. 38-50. Springer, Heidelberg (2012)

[2] Valente, M.: Demystifying the struggles of private sector paradigmatic change: Business as an agent in a complex adaptive system. Business \& Society 49, 439-476 (2010)

[3] Engler, J., Kusiak, A.: Modeling an Innovation Ecosystem with Adaptive Agents. International Journal of Innovation Science 3(2) (2011)

[4] Ramanathan, J.: Fractal architecture for the adaptive complex enterprise. Comm. of ACM 48, 51-57 (2005)

[5] Bider, I., Bellinger, G., Perjons, E.: Modeling an Agile Enterprise: Reconciling Systems and Process Thinking. In: Johannesson, P., Krogstie, J., Opdahl, A.L. (eds.) PoEM 2011. LNBIP, vol. 92, pp. 238-252. Springer, Heidelberg (2011)

[6] McQueen, P.: Physics and fractal structures. Journal of Statistical Physics 86, 1397-1398 (1997)

[7] Peffers, K., Tuunanen, T., Rothenberger, M.A., Chatterjee, S.: Design Science Research Methodology for Information Systems Research. Journal of Management Information Systems 24(3), 45-78 (2007)

[8] Bider, I., Johannesson, P., Perjons, E.: Design science research as movement between individual and generic situation-problem-solution spaces. In: Baskerville, R., De Marco, M., Spagnoletti, P. (eds.) Designing Organizational Systems: An Interdisciplinary Discourse. Springer (2012)

[9] Lawson, H.W.: A Journey Through the Systems Landscape. College Publications (2010)

[10] Hammer, M., Stanton, S.: How Process Enterprises Really Work. Harvard Business Review 77(6), 108-118 (1999)

[11] Scheer, A.-W.: ARIS - Business Process Modeling. Springer (2000)

[12] Elliott, B., Elliott, J.: Financial Accounting and Reporting. Financial Times/Prentice-Hall, London (2004)

[13] Hoverstadt, P.: The Fractal Oragnization: Creating Sustainable Oragnization with the Viable System Model. John Wiley \& Son (2008)

[14] Sandkuhl, K., Kirikova, M.: Analysing Enterprise Models from a Fractal Organisation Perspective - Potentials and Limitations. In: Johannesson, P., Krogstie, J., Opdahl, A.L. (eds.) PoEM 2011. LNBIP, vol. 92, pp. 193-207. Springer, Heidelberg (2011)

[15] Mercedes Canavesio, M., Martinez, E.: Enterprise modeling of a project-oriented fractal company for SMEs networking. Computers in Industry 58, 794-813 (2007)

[16] Shahzad, K., Elias, M., Johannesson, P.: Requirements for a Business Process Model Repository: A Stakeholders' Perspective. In: Abramowicz, W., Tolksdorf, R. (eds.) BIS 2010. LNBIP, vol. 47, pp. 158-170. Springer, Heidelberg (2010)

[17] Elias, M., Shahzad, K., Johannesson, P.: A Business Process Metadata Model for a Process Model Repository. In: Bider, I., Halpin, T., Krogstie, J., Nurcan, S., Proper, E., Schmidt, R., Ukor, R. (eds.) BPMDS 2010 and EMMSAD 2010. LNBIP, vol. 50, pp. 287-300. Springer, Heidelberg (2010) 